\title{
Prevalência de Anemia e Seus Fatores Determinantes em Gestantes de Município do Noroeste do Estado do RS
}

\author{
Giordana Gabrielle Frick ${ }^{1}$, Matias Nunes Frizzo ${ }^{2}$
}

\begin{abstract}
RESUMO
A anemia afeta em todo mundo 1,62 bilhão de pessoas, sendo as gestantes e crianças alguns dos grupos de populações mais acometidos. Este estudo teve por objetivo determinar a prevalência de anemia e seus fatores determinantes em gestantes. Foram analisados os dados de Hemoglobina (Hb), Hematócrito (Ht) e Volume Corpuscular Médio (VCM) de 107 gestantes, contudo apenas 77 delas foram entrevistadas, pelo fato de 3 gestações resultarem em aborto e de 27 não residirem mais no município, para definir as características e os fatores determinantes no desenvolvimento de anemia. Encontrou-se $14 \%(n=15)$ gestantes com Hb inferior a $11 \mathrm{~g} / \mathrm{dL}$, entre elas, $3 \%(n=3)$ com $\mathrm{Ht}$ inferior a $33 \%$. Ainda, $14 \%(n=15)$ gestantes possuíam VCM inferiores a $80 \mathrm{fL}$. Entre as gestantes entrevistadas, $16 \%(n=12)$ apresentaram anemia, e destas $67 \%(n=8)$ possuíam renda inferior a dois salários mínimos, $75 \%(n=9)$ relataram ter como grau de instrução o Ensino Fundamental e $25 \%$ ( $n=3$ ) consumiam poucos alimentos contendo ferro e não utilizavam sulfato ferroso como suplementação. A prevalência de anemia encontrada foi de $14 \%$ nas 107 gestantes avaliadas, estando esse número diretamente relacionado às condições socioeconômicas das participantes, o que refletiu no conhecimento da patologia, assim como na dieta ao longo da gestação. Este índice serve de um importante indicativo para a realização de medidas que possibilitem identificar, prevenir e orientar essas gestantes.
\end{abstract}

Palavras-chave: Gestantes. Hemograma. Anemia. Deficiência de ferro. Saúde pública.

PREVALENCE OF ANEMIA AND ITS DETERMINANTS FACTORS OF PREGNANT WOMEN NORTHWEST MUNICIPAL IN THE RS STATE

\section{ABSTRACT}

Anemia affects everyone 1.62 billion people, pregnant women and children and some of the population groups most affected. This study proposes to determine the prevalence of anemia and its determinants in pregnant women. Were analyzed for hemoglobin $(\mathrm{Hb})$, hematocrit $(\mathrm{Ht})$ and mean corpuscular volume (MCV) of 107 pregnant women, with only 77 of them were interviewed, due to the fact that 3 resulted in abortion and 27 did not reside in the municipality, to determine the characteristics and determinants in the development of anemia. It was found $14 \%(n=15)$ pregnant women with $\mathrm{Hb}$ less than $11 \mathrm{~g} /$ $\mathrm{dL}$, among them, three $3 \%(\mathrm{n}=3)$ with $\mathrm{Ht}$ less than $33 \%$. Still, $14 \%(\mathrm{n}=15)$ patients had MCV below $80 \mathrm{fL}$. Among the pregnant women interviewed, $16 \%(n=12)$ had anemia, these eight $67 \%(n=8)$ with incomes less than two minimum wages, $75 \%(n=9)$ reported as educated elementary school and $25 \%(n=3)$ consumed fewer foods containing iron and non ferrous sulfate used as a supplement. The prevalence of anemia was $14 \%$ in the 107 pregnant women evaluated. This number being directly related to the socioeconomic conditions of the pregnant women, which reflected in the knowledge of the pathology as well as in the diet during pregnancy. This index serves as an important indicator for the accomplishment of measures that make it possible to identify, prevent, and guide these pregnant women.

Keywords: Pregnant women. Blood cell count. Anemia. Iron deficiency. Public health.

Recebido em: $25 / 6 / 2015$

Aceito em: $13 / 7 / 2017$

\footnotetext{
Farmacêutica. Universidade Regional do Noroeste do Estado do Rio Grande do Sul - Unijuí. Especialista em Farmacologia e Interações Medicamentosas Uninter. Farmacêutica da Secretaria Municipal de Saúde de Cruz Alta - RS. giordana.frick@hotmail.com

${ }^{2}$ Farmacêutico. Doutor em Biologia Celular e Molecular. Professor-adjunto do Departamento de Ciências da Vida da Universidade Regional do Noroeste do Estado do Rio Grande do Sul e professor titular no Curso de Biomedicina na Faculdade CNEC Santo Ângelo. mnfrizzo@hotmail.com
} 


\section{INTRODUÇÃO}

A anemia é considerada a doença mais prevalente em todo o mundo e representa um quadro clínico importante, pois está associada a inúmeras etiologias, entre elas carência de elementos essenciais como ferro, ácido fólico e vitamina $B_{12}$ (TIMM, 2005). É determinada por um estado em que a concentração de hemoglobina no sangue torna-se baixa devido à carência de um ou mais dos nutrientes essenciais (ORGANIZAÇÃO..., 1968).

Estima-se que a anemia afeta 1,62 bilhão de pessoas no mundo, o que corresponde a $24,8 \%$ da população mundial, acometendo todas as fases da vida, contudo é mais prevalente nos grupos de populações gestantes (42\%) e crianças na pré-escola (47\%) (WORLD..., 2008).

Como a gestação é um fenômeno fisiológico, na maioria das vezes sua evolução ocorre normalmente, sem apresentar variações. Há, porém, uma parcela dessas mulheres que, por portarem características específicas ou por sofrerem algum agravo, apresentam maiores probabilidades de evolução desfavorável, tanto para o feto como para a mãe (BRASIL, 2001). Um exemplo disso é a carência de ferro, que resulta em anemia ferropênica, e apresenta alta prevalência entre gestantes (TIMM, 2005), pois nestas, em um curto período de tempo ocorre um aumento significativo na demanda de ferro, e o organismo reduz muito suas reservas, resultando em processo anêmico (WORLD..., 2001).

De acordo com a World Health Organization, estima-se que pelo menos $22 \%$ das gestantes de países industrializados apresentam deficiência de ferro. Já em países não industrializados, como é o caso do Brasil, calcula-se que $54 \%$ das gestantes apresentam quadro clínico de anemia, com a prevalência na deficiência de ferro variando de acordo com fatores relacionados ao indivíduo, como idade, sexo, condições fisiológicas, patológicas e ambientais (WORLD..., 2008). Ainda, quanto à condição anêmica na gestação, associa-se os fatores de baixo nível socioeconômico, reservas maternas inadequadas de ferro, ausência de suplementação de ferro e dietas deficientes do mineral. Desse modo, a anemia correlacionada aos fatores de risco desencadeia uma série de repercussões materno-fetais, tornando-a um problema de saúde pública (OLIVEIRA; BARROS; FERREIRA, 2015).

Diversos parâmetros podem ser avaliados na gestação, entre eles o Hematócrito $(\mathrm{Ht})$ e a Hemoglobina $(\mathrm{Hb})$, conforme Dani et al. (2008), mas também se ressalta a importância clínica do Volume Corpuscular Médio (VCM), pois representa o valor médio do "tamanho" de hemácias que realmente foram avaliadas e que após o processo de automação passou a ser mais confiável (MOREIRA; NOVAIS; NASCIMENTO, 2005). Nas anemias ferropênicas, o principal achado laboratorial associado ao quadro anêmico é a redução do VCM, o qual é reportado como microcitose, portanto quadros anêmicos associados à microcitose são geralmente relacionados à redução dos estoques de ferro corporais, como acontece durante a gestação (CARVALHO; BARACAT; SGARBIERI, 2006).

Para definir as condições de anemia nas gestantes considera-se os valores de hemoglobina menores que $11 \mathrm{mg} / \mathrm{dl}$ (LOPES; FREITAS; MACIEL, 2015). Além disso, a deficiência grave de ferro é bastante evidente, pois causa anemia microcítica e hipocrômica. E para o diagnóstico específico é importante avaliar os parâmetros como VCM, em que valores menores que $80 \mathrm{fL}$ é considerado microcitose e valores entre 80 e $96 \mathrm{fL}$, como normocíticas. Ainda, correlacionar com a amplitude de variação do tamanho dos eritrócitos ou red distribution width (RDW), e com a hipocromia, considerada, quando o índice de Hemoglobina Corpuscular Média (HCM), que avalia a concentração de hemoglobina no eritrócito, for menor que $27 \mathrm{pg}$ (TAPIA et al., 2010).

Durante o período gestacional ocorrem mudanças no organismo, como no útero, que sofre modificações de hipertrofia e dilatação, requerendo maior perfusão sanguínea, como consequência aumentando a vascularização. Assim, podem surgir ou já estarem instalados problemas na gestação, tais como: aborto espontâneo, partos prematuros, hemorragias durante o trabalho de parto, hipóxia, recém-nascido de baixo peso, ou com anemia e baixa na imunidade (LOPES; FREITAS; MACIEL, 2015).

Dessa forma, o presente estudo tem como objetivo determinar a prevalência de anemia e seus fatores determinantes em gestantes em um município do Noroeste do Estado do Rio Grande do Sul no período de abril de 2009 a abril de 2011.

\section{MATERIAS E MÉTODOS}

Trata-se de um estudo de levantamento de dados acerca dos hemogramas das gestantes de um município da região Noroeste do Estado do Rio Grande do Sul. Após a coleta dos resultados dos hemogramas foram realizadas entrevistas com as participantes por meio de instrumento padronizado, questionário elaborado pelos pesquisadores, com caráter analítico e retrospectivo aprovado pelo Comitê de Ética em Pesquisa da Universidade Regional do Noroeste do Esta- 
do do Rio Grande do Sul - CEP/Unijuí - sob o Parecer Consubstanciado no 161/2011. O estudo foi desenvolvido com gestantes que fizeram acompanhamento pré-natal, no primeiro trimestre de gestação, em Unidade Básica de Saúde de um município do Noroeste do Estado do Rio Grande do Sul no período de 1으 de abril de 2009 a 30 de abril de 2011 e que realizaram o hemograma em Laboratório de Análises Clínicas do município.

A amostra constitui-se de 107 gestantes, porém a entrevista foi realizada com apenas 77 mulheres, pelo fato de 3 gestações resultarem em aborto e de 27 não residirem mais no município. Todas as mulheres participantes do estudo assinaram voluntariamente o Termo de Consentimento Livre e Esclarecido e responderam aos questionamentos dos pesquisadores.

A coleta de dados foi realizada nos meses de juIho e agosto de 2011 e para tanto fez-se uso de um instrumento validado, a partir do qual foi possível obter informações que definiram as características da gestante e os fatores determinantes para o desenvolvimento de anemia, tais como idade, escolaridade, profissão, renda, antecedentes pessoais de anemia, alimentação rica em ferro e suplementação medicamentosa com ferro.

Nos dados do eritrograma do primeiro trimestre de gestação de cada mulher obtidos em laboratório do município em estudo, foram analisados os valores de Hemoglobina $(\mathrm{Hb})$, Hematócrito $(\mathrm{Ht})$ e Volume Corpuscular Médio (VCM), os quais definiram se houve presença ou não de anemia e auxiliaram na sua classificação. Foram consideradas anêmicas as gestantes que apresentaram $\mathrm{Hb}$ inferior a $11 \mathrm{~g} / \mathrm{dL}$ e $\mathrm{Ht}$ menor do que 33\% e classificadas como hemácias microcíticas os resultados de VCM menores do que $80 \mathrm{fL}$ e normocíticas entre 80 e $100 \mathrm{fL}$, tendo como base os valores de referência do laboratório que realizou as análises.

Os resultados de cada paciente foram associados às respostas obtidas na entrevista, analisados de forma descritiva e matemático-estatística, utilizando frequência absoluta e relativa.

\section{RESULTADOS}

Com relação aos 107 hemogramas avaliados das gestantes atendidas na rede pública, encontrou-se $14 \%(n=15)$ com $\mathrm{Hb}$ inferior a $11 \mathrm{~g} / \mathrm{dL}$. Entre estas, $3 \%(n=3)$ apresentaram $\mathrm{Ht}$ inferior a 33\%. Avaliando-se o VCM, verificou-se que $14 \%(n=15)$ gestantes possuíam valores inferiores a $80 \mathrm{fL}$; apenas $7 \%(n=7)$ dos hemogramas das gestantes encontravam-se com ane- mia microcítica e $53 \%(n=8)$ com anemia normocítica. Ainda pode-se afirmar que da totalidade de anemias encontradas, $47 \%$ são microcíticas e $53 \%$ são normocíticas. Esses resultados estão ilustrados na Tabela $1 \mathrm{e}$ na Figura 1.

Tabela 1 - Índices hematimétricos distribuídos acima e abaixo dos valores de referência para gestantes, ljuí-RS/2011

\begin{tabular}{lrr}
\hline \multicolumn{1}{c}{ Índices } & $\mathbf{N}$ & $\%$ \\
$\mathrm{Hb}<11 \mathrm{~g} / \mathrm{dL}$ & 15 & 14,02 \\
$\mathrm{Hb}>11 \mathrm{~g} / \mathrm{dL}$ & 92 & 85,98 \\
\hline Total & 107 & 100,00 \\
\cline { 1 - 2 } $\mathrm{Ht}<33 \%$ & 3 & 2,80 \\
$\mathrm{Ht}>33 \%$ & 104 & 97,20 \\
\hline Total & 107 & 100,00 \\
\hline $\mathrm{VCM}<80 \mathrm{fL}$ & 15 & 14,02 \\
$\mathrm{VCM}>80 \mathrm{fL}$ & 92 & 85,98 \\
Total & 107 & 100,00 \\
$\mathrm{Hb}<11 \mathrm{~g} / \mathrm{dL}$ e $V C M<80 \mathrm{fL}$ & 7 & 6,54 \\
$\mathrm{Hb}<11 \mathrm{~g} / \mathrm{dL}$ e $V C M>80 \mathrm{fL}$ & 8 & 7,48 \\
$\mathrm{Hb}>11 \mathrm{~g} / \mathrm{dL}$ e $V C M<80 \mathrm{fL}$ & 8 & 7,48 \\
$\mathrm{Hb}>11 \mathrm{~g} / \mathrm{dL}$ e $V C M>80 \mathrm{fL}$ & 84 & 78,50 \\
\hline Total & 107 & 100,00 \\
\hline
\end{tabular}

Figura 1 - Classificação das anemias conforme o valor do VCM, Ijuí-RS/2011

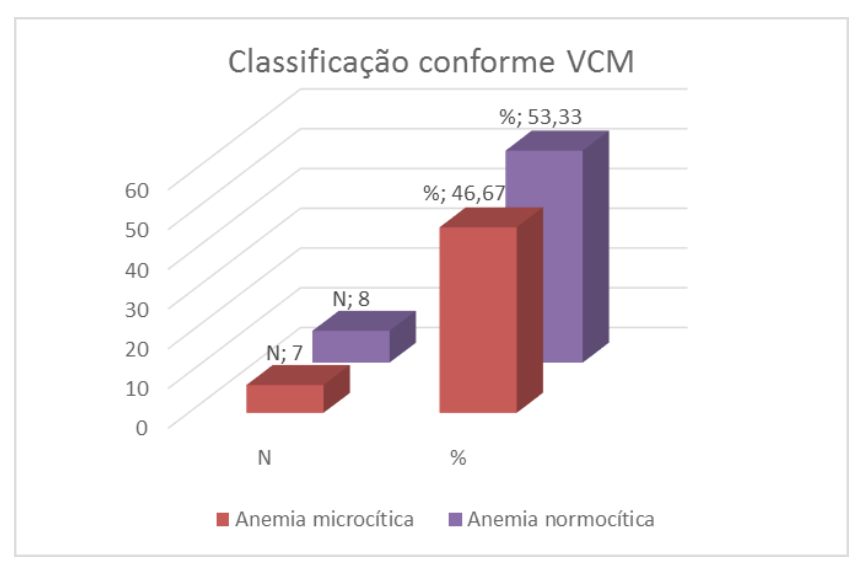

Após a avaliação dos 107 hemogramas, somente 77 gestantes foram entrevistadas, destas, $26 \%$ $(n=20)$ relataram ter histórico de anemia. Entre as muIheres que descreverem possuir um histórico de anemia $13 \%(n=10)$ apresentavam anemia e $6 \%(n=5)$ possuíam microcitose. Somente $4 \%(n=3)$ das gestantes entrevistadas tinham histórico para anemia, anemia e microcitose associados. Esses dados estão demonstrados na Tabela 2. 
Tabela 2 - Distribuição das gestantes entrevistadas de acordo com a presença ou não de anemia, história prévia de anemia e microcitose, ljuí-RS/2011

\begin{tabular}{c|c|c|c|c|c|c}
\hline \multirow{2}{*}{ Variável } & \multicolumn{2}{|c|}{$\begin{array}{c}\text { Histórico de } \\
\text { Anemia }\end{array}$} & \multicolumn{2}{c|}{ Anêmicas } & \multicolumn{2}{c}{ Microcitose } \\
\cline { 2 - 7 } & $\mathbf{n}$ & $\mathbf{\%}$ & $\mathbf{n}$ & $\mathbf{\%}$ & $\mathbf{n}$ & $\mathbf{\%}$ \\
\hline \multirow{2}{*}{ SIM } & 20 & 25,97 & 10 & 12,99 & 5 & 6,49 \\
\hline NÃO & 57 & 74,03 & 67 & 87,01 & 72 & 93,51 \\
\hline Total & $\mathbf{7 7}$ & $\mathbf{1 0 0 , 0 0}$ & $\mathbf{7 7}$ & $\mathbf{1 0 0 , 0 0}$ & $\mathbf{7 7}$ & $\mathbf{1 0 0 , 0 0}$ \\
\hline
\end{tabular}

Observa-se que a faixa etária das 77 gestantes entrevistadas variou entre 10 e 49 anos, e a maioria delas, $44 \%(n=34)$, apresentam idade entre $20-29$ anos e destas apenas $26 \%(n=20)$ relataram estar trabalhando. A maioria das gestantes, $58 \%(n=45)$, referiram ter estudado até o Ensino Fundamental e $42 \%$ $(n=32)$ mencionaram ter cursado Ensino Médio. Além disso, as gestantes da faixa etária de 20-29 anos são as que apresentam o maior nível de escolaridade e a maior proporção de trabalho. Observar Tabela 3.

Na Tabela 4 é possível observar que a maioria das gestantes $79 \%(n=61)$ possui renda familiar inferior a 2 salários mínimos mensais, e destas, apenas $14 \%(n=11)$ relataram ingerir muitos alimentos ricos em ferro no período de gestação. Ainda, entre as gestantes com baixa renda, $18 \%$ ( $n=14$ ) não faziam suplementação medicamentosa de ferro.

Tabela 3 - Distribuição das gestantes entrevistadas conforme idade, correlacionando com escolaridade e trabalho, ljuí-RS/2011

\begin{tabular}{|c|c|c|c|c|c|c|c|c|c|c|}
\hline \multirow{2}{*}{\multicolumn{3}{|c|}{ Faixa etária (em anos) }} & \multicolumn{4}{|c|}{ Escolaridade } & \multicolumn{4}{|c|}{ Trabalha } \\
\hline & & & \multicolumn{2}{|c|}{$\begin{array}{c}\text { Ensino } \\
\text { Fundamental }\end{array}$} & \multicolumn{2}{|c|}{$\begin{array}{l}\text { Ensino } \\
\text { Médio }\end{array}$} & \multicolumn{2}{|c|}{ Sim } & \multicolumn{2}{|c|}{ Não } \\
\hline Intervalo & $n$ & $\%$ & $n$ & $\%$ & $\mathbf{n}$ & $\%$ & $\mathbf{n}$ & $\%$ & $\mathrm{n}$ & $\%$ \\
\hline $10-19$ & 20 & 25,97 & 14 & 18,18 & 6 & 7,79 & 8 & 10,39 & 12 & 15,59 \\
\hline $20-29$ & 34 & 44,16 & 17 & 22,08 & 17 & 22,08 & 20 & 25,97 & 14 & 18,18 \\
\hline $30-39$ & 22 & 28,57 & 13 & 16,88 & 9 & 11,69 & 16 & 20,78 & 6 & 7,79 \\
\hline $40-49$ & 1 & 1,30 & 1 & 1,30 & 0 & 0,00 & 1 & 1,30 & 0 & 0,00 \\
\hline Total & 77 & 100,00 & 45 & 58,44 & 32 & 41,56 & 45 & 58,44 & 32 & 41,56 \\
\hline
\end{tabular}

Ao se tratar da profissão descrita pelas entrevistadas, a maioria 39\% ( $n=30$ ) relatou ser agricultora, seguida de $38 \%$ ( $n=29$ ) com atividades do lar. Os dados estão explanados na Figura a seguir.

Figura 2 - Distribuição da atividade profissional das gestantes, ljuí-RS/2011

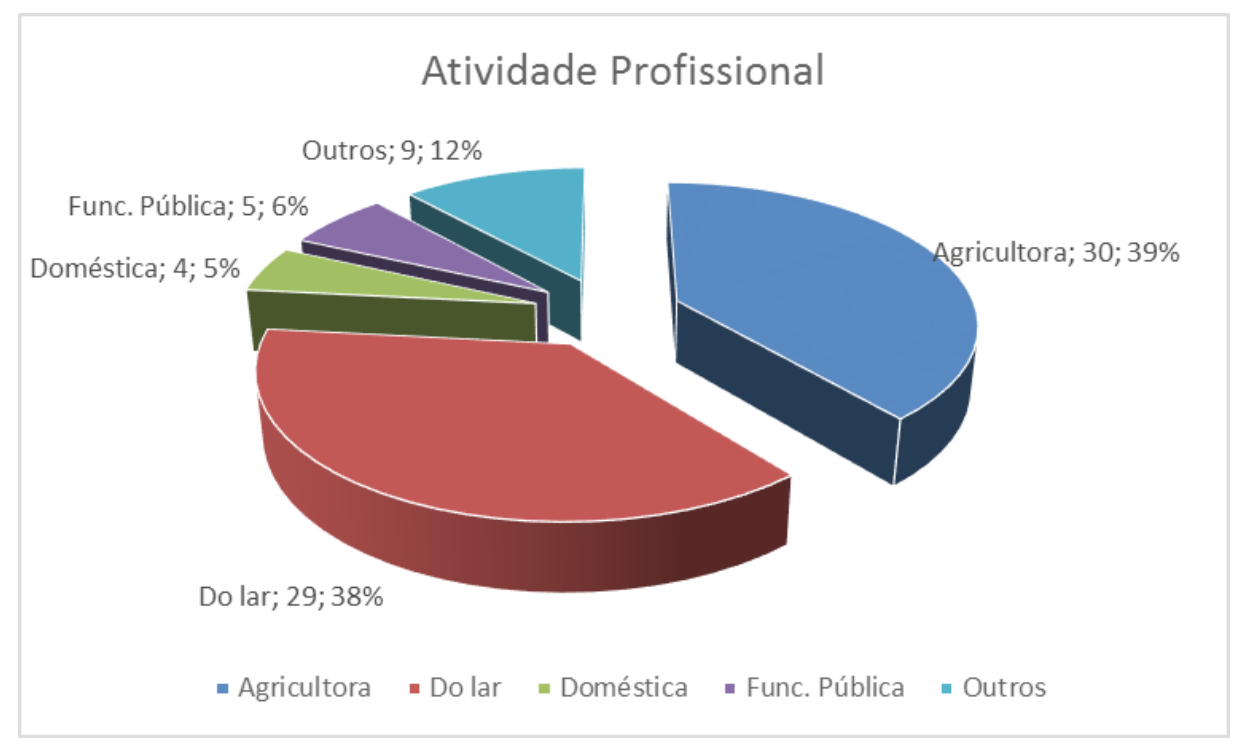


Tabela 4 - Nível socioeconômico das gestantes relacionado com a ingestão de ferro por meio da alimentação e suplementação medicamentosa, ljuí-RS/2011

\begin{tabular}{|c|c|c|c|c|c|c|c|c|c|c|c|c|}
\hline \multirow{2}{*}{\multicolumn{3}{|c|}{$\begin{array}{l}\text { Renda Familiar (em salá- } \\
\text { rios mínimos) }\end{array}$}} & \multicolumn{6}{|c|}{ Alimentos ricos ferro } & \multicolumn{4}{|c|}{ Sulfato ferroso } \\
\hline & & & \multicolumn{2}{|c|}{$\begin{array}{c}\text { Raros } \\
\text { (1 tipo) }\end{array}$} & \multicolumn{2}{|c|}{$\begin{array}{c}\text { Poucos } \\
\text { ( } 2 \text { tipos) }\end{array}$} & \multicolumn{2}{|c|}{$\begin{array}{c}\text { Muitos } \\
\text { (3 ou mais) }\end{array}$} & \multicolumn{2}{|l|}{ SIM } & \multicolumn{2}{|c|}{ Não } \\
\hline & $\mathrm{n}$ & $\%$ & $\mathbf{n}$ & $\%$ & $\mathbf{n}$ & $\%$ & $\mathrm{n}$ & $\%$ & $\mathbf{n}$ & $\%$ & $\mathrm{n}$ & $\%$ \\
\hline $\begin{array}{c}\text { Menos } \\
\text { de } 2\end{array}$ & 61 & 79,22 & 12 & 15,58 & 38 & 49,35 & 11 & 14,29 & 47 & 61,04 & 14 & 18,18 \\
\hline $\begin{array}{c}\text { Mais de } \\
2\end{array}$ & 16 & 20,78 & 2 & 2,60 & 6 & 7,79 & 8 & 10,39 & 7 & 9,09 & 9 & 11,69 \\
\hline Total & 77 & 100,00 & 14 & 18,18 & 44 & 57,14 & 19 & 24,68 & 54 & 70,13 & 23 & 29,87 \\
\hline
\end{tabular}

Entre as gestantes entrevistadas, $16 \%(n=12)$ apresentaram anemia, destas $67 \%(n=8)$ possuíam renda de até dois salários mínimos e $33 \%(n=4)$ renda superior a dois salários mínimos. Abordando a questão da escolaridade das gestantes anêmicas entrevistadas $75 \%(n=9)$ relataram ter como grau de instrução o Ensino Fundamental e $25 \%(n=3)$ o Ensino Médio. Além disso, das $16 \%(n=12)$ gestantes anêmicas entrevistadas, apenas $17 \%(n=2)$ descreveram ingerir muitos alimentos ricos em ferro e fazer uso de suplementação medicamentosa durante a gestação; $50 \%(n=6)$ relataram ingerir poucos alimentos contendo ferro, porém faziam uso de suplementação medicamentosa. Já $25 \%(n=3)$ e $8 \%(n=1)$ consumiam respectivamente, raros e poucos alimentos contendo ferro e não utilizavam sulfato ferroso como suplementação.

\section{DISCUSSÃO}

Entre as várias metodologias que podem ser usadas para determinação da anemia, o presente estudo definiu como parâmetro $\mathrm{Hb}$ inferior a $11 \mathrm{~g} / \mathrm{dL}$ e $\mathrm{Ht}$ inferior a $33 \%$, que esteve presente em $14 \%$ dos 107 hemogramas avaliados, descrevendo uma prevalência de $14 \%$ na população avaliada. Resultado semelhante foi encontrado no estudo de Araújo et al. (2013), no qual aproximadamente $10 \%$ das gestantes, no primeiro trimestre de gestação, caracterizaram-se anêmicas. Já Fujimori et al. (2009) analisaram a prevalência de anemia em gestantes de regiões distintas, e encontraram um valor mais acentuado, de $25,5 \%$ e $10,6 \%$ respectivamente, nas regiões Centro-Oeste e Sul do Brasil. Essa diferença na incidência de anemia deve-se a características que interferem no quadro da doença, como maior proporção de gestantes adolescentes, com baixo peso, com abortamentos e mais de duas gestações anteriores. Já no presente estudo pode-se afirmar que a prevalência de baixa renda nas gestantes com anemia e a pouca ingestão de alimentos ricos em ferro interferiram no quadro da doença.

Sabe-se que a anemia por deficiência de ferro durante a gravidez aumenta os riscos de mortalidade materna, riscos de mortalidade materna, perinatal e infantil. Estima-se que $40 \%$ de todas as mortes maternas perinatais estão ligadas à anemia. Além disso, os filhos de mães anêmicas têm menos da metade das reservas de ferro normal, necessitando de mais ferro do que é fornecido pelo leite materno, em relação ao bebê de peso normal; além disso, também causa consequências a longo prazo no desenvolvimento mental (WORLD..., 2001). Por isso, existe a necessidade de reforçar programas educacionais enfatizando os riscos e problemas que podem ocorrer durante a gravidez (TAPIA et al., 2010). Ressalta-se também, por outro lado, a importância da atuação das equipes multiprofissionais de saúde no acompanhamento, na assistência e na orientação mais direcionada e frequente às gestantes com anemias e aos seus respectivos filhos, nos primeiros meses de vida.

Outro parâmetro a ser considerado é o Volume Corpuscular Médio (VCM), que determina, nesse caso, se as hemácias estão microcíticas ou não. Verificou-se que esta alteração também esteve presente em um número significativo de gestantes, porém destas com microcitose, um percentual considerável também possuía anemia. Comparando-se ao estudo de Tapia et al. (2010), no qual foram analisados os índices hematimétricos, observou-se que $26 \%(n=31)$ gestantes anêmicas apresentaram microcitose. Já para Santos et al. (2009), foram $40 \%(n=8)$ as gestantes que possuíam 
anemia e microcitose. Observou-se, então, que o índice de anemias microcíticas é elevado, uma vez que $46,67 \%$ das anemias nas gestantes foram microcíticas.

Comparando estes resultados com os demais estudos, verifica-se uma similaridade na incidência de anemias microcíticas, pois, segundo Olivares e Walter (2004), como a maior frequência de anemia na gestação é devido à deficiência de ferro, inicialmente os primeiros depósitos do organismo são esgotados podendo se caracterizar como anemia normocítica, porém se ainda há ingestão insuficiente, esta pode evoluir para um estado mais grave de anemia, que é microcítica. Para Tapia et al. (2010), contudo, outros fatores também podem induzir a uma anemia microcítica, entre eles doenças infecciosas diversas, perdas por hemorragias ou redução da absorção de ferro ou mesmo doenças genéticas, como as talassemias. Cabe salientar que muitas gestantes com microcitose podem estar sem anemia, mas já estão numa fase de depleção de ferro. Isso ocorre devido à redução dos estoques de ferro que pode levar a quadros de microcitose sem a presença de anemia (GROTTO, 2010).

Conforme demonstram os resultados, apesar dos índices elevados de anemias microcíticas, ainda houve predominância de anemias normocíticas, que consistem na deficiência concomitante de ferro, mas este processo deve-se a um quadro inicial de anemia em que ainda não se deflagrou um quadro de anemia e microcitose. Ou também é possível correlacionar a presença de anemia associada à carência de ferro, vitamina B12 e ácido fólico, os quais podem gerar um quadro anêmico normocítico.

Outra questão preocupante é em relação ao número de gestantes anêmicas e com história prévia de anemia - $13 \%$ ( $n=10)$ - ressaltando-se que essa carência pode ser relacionada com a alimentação pobre em ferro consumida por essas mulheres antes mesmo da gestação, e também devido a fatores genéticos, como o traço talassêmico, que segundo Cançado (2006), pode ser denominado de portador silencioso, e resulta em uma forma de talassemia praticamente assintomática e com alterações laboratoriais mínimas ou ausentes, o que dificulta o seu diagnóstico por técnicas laboratoriais convencionais. Diante deste caso, é notório que a prevenção das anemias hereditárias deva começar o mais precocemente possível e ser feita por meio de ações educadoras, de um diagnóstico laboratorial realizado por profissionais capacitados e por estudo familiar (SAKAMOTO et al., 2008).

Em relação às variáveis socioeconômicas e demográficas, verifica-se que houve predominância de mulheres gestantes com idade entre 20-29 anos de idade, que referiram ter estudado até o Ensino Fundamental e que trabalhavam. Em outro estudo, Sato et al. (2008) analisaram uma amostra composta por 750 gestantes, e destas $64 \%(n=482)$ tinham idade entre 20-30 anos, 34\% ( $n=253$ ) estudaram até o Ensino Fundamental e $48,5 \%$ trabalhavam. Segundo o Programa das Nações Unidas para o Desenvolvimento - PNUD (Brasil, 2017), o nível de escolaridade da mãe influencia decisivamente desde a gravidez e até na mortalidade infantil, destacando-se que o município estudado tem um índice de escolaridade muito baixo (média de 4 anos) e de mortalidade considerável, uma vez que a taxa de mortalidade infantil média na cidade foi de 18,87 para 1.000 nascidos vivos, em 2010, o que justifica os resultados encontrados nessa pesquisa e reforça a necessidade de uma atenção especial para gestantes e filhos, por intermédio das equipes de saúde.

Em relação à renda familiar das gestantes, o resultado encontrado no estudo de Oliveira, Barros e Ferreira (2015) mostrou-se similar ao obtido nesta pesquisa, constatando-se que quase $52 \%(n=212)$ apresentavam renda familiar mensal de um a dois salários mínimos e 79\% ( $n=61)$ relataram não atingir dois salários mínimos mensais, respectivamente. Essa prevalência de baixa renda nas mulheres pode justificar a pouca ingestão de alimentos ricos em ferro durante a gestação, mesmo a maioria delas $39 \%(n=30)$ sendo agricultoras, e também os casos de anemias encontrados. Aliás, essa prevalência de baixa renda, segundo o Instituto Brasileiro de Geografia e Estatística -IBGE - (2017), é explicada por dados do ano de 2010, que revelaram um índice elevado de pobreza e de desigualdade no município estudado, no qual cerca de $46 \%$ da população possuía renda de até meio salário mínimo por pessoa.

Salienta-se ainda que mulheres com maior escolaridade e integrantes de classes econômicas mais altas têm chances significativamente superiores de realizar pelo menos seis consultas de pré-natal e a consulta de puerpério do que aquelas em situação mais desfavorável (BRASIL, 2009).

Entre as 12 gestantes anêmicas entrevistadas, pôde-se observar que $25 \%$ ( $n=3$ ) não utilizavam medicamento como suplementação e ainda consumiam raros alimentos contendo ferro, o que difere do encontrado por Oliveira, Barros e Ferreira (2015), em que quase $100 \%$ das gestantes, incluindo aquelas anêmicas, apresentaram um inadequado consumo de ferro alimentar. Obviamente, segundo Gillespie, Kevany e Mason (1991), a fortificação de alimentos não substitui necessariamente a suplementação com ferro, mas se efetiva a longo prazo, podendo aumentar as reservas desse mi- 
neral no organismo. Pode-se observar ainda que apesar dos índices de gestantes anêmicas não serem elevados, a carência nutricional é notada mesmo quando essas gestantes ainda não se apresentam anêmicas segundo valores de $\mathrm{Hb}$ e $\mathrm{Ht}$ e por meio das reduções nos valores de ferritina, por exemplo (DANI et al., 2008).

As mulheres gestantes são um grupo prioritário no que se refere à suplementação de ferro, porém no estudo um número significativo, $30 \%(n=23)$, não fazia uso dessa suplementação. Sabe-se que além da ingestão regular de alimentos contendo esse elemento, é essencial e de competência dos serviços de saúde primários a distribuição de comprimidos orais de ferro a todas as gestantes, haja vista que essa medida preventiva tem grande chance de sucesso quando dirigida a grupos específicos vulneráveis, como é o caso das gestantes. Além disso, devido ao seu baixo custo e alta biodisponibilidade, o sulfato ferroso é o fármaco de escolha nesses casos (GILLESPIE; KEVANY; MASON, 1991).

Ademais, percebe-se que o uso do sulfato ferroso, mesmo em mulheres que não apresentam anemia, como profilaxia, é imprescindível para diminuir o desenvolvimento de anemias, hemorragias pós-parto, subnutrição em feto, que pode aumentar a morbimortalidade tanto materna quando fetal (LOPES; FREITAS; MACIEL, 2015).

Para Lopes, Freitas e Maciel (2015), os processos que desencadeiam anemia por deficiência de ferro, bem como o desconhecimento das gestantes acerca da prevenção dessa patologia, influenciam diretamente na manifestação da anemia ferropênica. Fica evidente que cada vez mais é necessário investir em pesquisas sobre como intervir nos atendimentos às gestantes visando à prevenção da anemia durante o pré-natal.

Outra limitação do estudo que pode justificar a baixa prevalência de anemia encontrada é o fato de se ter analisado somente os hemogramas do período inicial da gestação, e não de todos os trimestres, uma vez que, conforme o estudo de Dani et al. (2008), a carência, geralmente, não está presente ao início da gestação, concretizando-se com os passar dos meses. Essa deficiência é causada pelo aumento das necessidades requeridas pelo feto ou pelo ajuste hemodinâmico que ocorre em todos os processos gestativos.

\section{CONCLUSÃO}

Os resultados deste estudo revelam que $14 \%$ das gestantes atendidas na Unidade Básica de Saúde do município em estudo estavam anêmicas, conside- rando que foram avaliados os dados somente do primeiro trimestre de gestação. Também ressalta-se os casos ocultos de anemia, que podem ser devido ao fato de várias gestantes apresentarem depleção de ferro. Sugere-se, ainda, um fundado suporte de diagnóstico laboratorial e ações educativas, visando a contribuir para a redução dos fatores determinantes que influem na anemia.

Verificou-se que fatores determinantes, como a limitada escolaridade, a reduzida renda familiar mensal e a pouca ingestão de alimentos ricos em ferro mostram ser este um grupo carente, e refletiram diretamente na predisposição do desenvolvimento de anemia nas gestantes e no feto, pois quanto menor o grau de instrução, menores são as oportunidades de emprego e de renda, com isso maior é a dificuldade de acesso aos alimentos e aos serviços de saúde. Não obstante, esses fatores servem como barreira quando há necessidade de adesão ao tratamento medicamentoso, pois mensuram a realidade da autopercepção de saúde.

Considerando o índice mundial e nacional, houve baixa prevalência de anemia no grupo de gestantes estudado, porém confirma-se que os fatores como renda, escolaridade, alimentação e suplementação medicamentosa estão diretamente ligados ao desenvolvimento da patologia. Desse modo, reforça-se a necessidade de priorizar programas assistenciais que possibilitem identificar, orientar, prevenir e dar assistência à saúde das gestantes.

\section{REFERÊNCIAS}

ARAÚJO, C. R. M. A. et al. Níveis de hemoglobina e prevalência de anemia em gestantes atendidas em unidades básicas de saúde, antes e após a fortificação das farinhas com ferro. Rev. Bras. Epidemiol., vol. 16, n. 2, p. 535-545, 2013. Disponível em: <http://www.scielosp.org/pdf/rbepid/ v16n2/1415-790X-rbepid-16-02-00535.pdf>. Acesso em: 10 ago. 2017.

BRASIL, Ministério da Saúde. Secretaria Executiva. Gestante de alto risco: Sistemas estaduais de referência hospitalar à gestante de alto risco. Brasília: Ministério da Saúde, 2001. Disponível em: <http://abenfo.redesindical.com.br/arqs/ outros/03052010/2001_gestantes.pdf>. Acesso em: 4 maio 2011.

Programa das Nações Unidas para o Desenvolvimento. Filhos de mães que estudam morrem menos. Brasília: PNUD, 2017.

- Ministério da Saúde. Pesquisa Nacional de Demografia e Saúde da Criança e da Mulher-PNDS 2006: dimensões do processo reprodutivo e da saúde da criança. Brasília: Ministério da Saúde, 2009. 
CANÇADO, R. D. Talassemias alfa. Rev. Bras. Hematol. Hemoter. vol. 28, n. 2, p. 81-87, 2006. Disponível em: <http://www.scielo.br/pdf/rbhh/v28n2/v28n2a05.pdf>. Acesso em: 8 ago. 2017.

CARVALHO, M. C.; BARACAT, E. C. E.; SGARBIERI, V. C. Anemia ferropriva e anemia de doença crônica: distúrbios do metabolismo de ferro. Segurança Alimentar e Nutricional, Campinas, vol. 13, n. 2, p. 54-63, 2006. Disponível em: <https://periodicos.sbu.unicamp.br/ojs/index.php/san/article/ view/1832>. Acesso em: 14 ago. 2017.

DANI, C. et al. Prevalência da anemia e deficiências nutricionais, através de diferentes parâmetros laboratoriais, em mulheres grávidas atendidas em dois serviços de saúde pública no Rio Grande do Sul. Rev. Bras. de Anál. Clín., vol. 40, n. 3, p. 171-175, 2008.

FUJIMORI, E. et al. Anemia em gestantes de municípios das Regiões Sul e Centro-Oeste do Brasil. Rev. Esc. Enferm., vol. 43, Esp. 2, p. 1.204-1.209, 2009. Disponível em: <http:// www.scielo.br/pdf/reeusp/v43nspe2/a10v43s2.pdf>. Acesso em: 9 ago. 2017.

GILLESPIE, S.; KEVANY, J.; MASON, J. Controlling Iron Deficiency: Nutrition policy discussion paper, n. 9. p. 1-74, fev. 1991. Disponível em: <http://www.unscn.org/layout/modules/resources/files/Policy_paper_No_9.pdf>. Acesso em: 9 ago. 2017.

GROTTO, H. Z. W. Diagnóstico laboratorial da deficiência de ferro. Rev. Bras. Hematol. Hemoter. vol. 32, Supl. 2, p. $22-$ 28, 2010. Disponível em: <http://www.scielo.br/pdf/rbhh/ v32s2/aop46010.pdf>. Acesso em: 9 ago. 2017.

INSTITUTO BRASILEIRO DE GEOGRAFIA E ESTATÍSTICA (IBGE). Informações estatísticas. 2017. Disponível em: <https://cidades.ibge.gov.br/v4/brasil/rs/redentora/panorama>. Acesso em: 8 ago. 2017.

LOPES, S. V. L. M.; FREITAS, I. R.; MACIEL, M. C. C. Anemia ferropriva/ferropênica em gestantes: uma revisão integrativa de literatura. Revista da Universidade Vale do Rio Verde, Três Corações, v. 13, n. 1, p. 442-451, 2015. Disponível em: <http://periodicos.unincor.br/index.php/revistaunincor/ article/viewFile/1989/pdf_320>. Acesso em: 10 ago. 2017.

MOREIRA, M. I. A.; NOVAIS, S. L.; NASCIMENTO, M. L. P. Freqüência de Anemias Microcíticas e Macrocíticas no Laboratório Dalmo Oliveira em Recife, PE. NewsLab, vol. 70 p. 91-96, 2005. Disponível em: <http://www.newslab.com.br/ ed_anteriores/70/art01.pdf>. Acesso em: 30 set. 2011.

OLIVARES, M.; WALTER, T. Causas y consecuencias de la deficiencia de hierro. Rev. Nutr., vol. 17, n. 1, p. 5-14, 2004. Disponível em: <http://www.scielo.br/pdf/rn/v17n1/ a01v17n1.pdf>. Acesso em: 9 ago. 2017.

OLIVEIRA, A. C. M.; BARROS, A. M. R.; FERREIRA, R. C. Fatores associados à anemia em gestantes da rede pública de saúde de uma capital do Nordeste do Brasil. Rev. Bras. Ginecol. Obstet., vol. 37, n. 11, p. 505-511, 2015. Disponível em: <http://www.scielo.br/pdf/rbgo/v37n11/0100-7203-rbgo-37-11-00505.pdf>. Acesso em: 8 ago. 2017.
ORGANIZAÇÃO MUNDIAL DE SAÚDE (OMS). Anemias Nutricionales. Séries de Informes Técnicos, Ginebra, n. 405, 1968. Disponível em: <http://whqlibdoc.who.int/trs/WHO_ TRS_405_spa.pdf>. Acesso em: 7 out. 2011.

SAKAMOTO, T. M. et al. Talassemia ß intermediária em gestante. Rev. Bras. Hematol. Hemoter., vol. 30, n. 6, p. 498500, 2008. Disponível em: <http://www.scielo.br/pdf/rbhh/ v30n6/v30n6a15.pdf>. Acesso em: 30 set. 2011.

SANTOS, T. F. et al. Avaliação do acompanhamento de gestantes com hemoglobina baixa em uma unidade básica de saúde do Estado de São Paulo. Revista Interdisciplinar de Estudos Experimentais, vol. 3, n. 1, p. 105-109, 2009. Disponível em: <http://www.editoraufjf.com.br/revista/index. php/riee/article/view/951/816>. Acesso em: 24 out. 2011.

SATO, A. P. S. et al. Prevalência de anemia em gestantes e a fortificação de farinhas com ferro. Texto Contexto Enferm., vol. 17, n. 3, p. 474-481, 2008. Disponível em: <http://www. scielo.br/pdf/tce/v17n3/a08v17n3.pdf>. Acesso em: 20 out. 2010.

TAPIA, M. E. L. et al. Prevalência de anemia nas gestantes atendidas no Sistema Único de Saúde - Secretaria Municipal de Saúde - Prefeitura de Belo Horizonte. RBAC, vol. 42, n. 4, p. 277-281, 2010. Disponível em: <http://www.sbac. org.br/pt/pdfs/rbac/rbac_42_04/rbac_42_04_10.pdf $>$. Acesso em: 26 out. 2011.

TIMM, G. Anemias: causas e implicações das alterações eritrocíticas. 2005. 52f. Monografia de conclusão de curso - Instituto de Biologia da Universidade Federal de Pelotas. Pelotas, RS, 2005. Disponível em: <http://www.ufpel.edu. $\mathrm{br} / \mathrm{prg} / \mathrm{sisbi} / \mathrm{bibct} / \mathrm{acervo} / \mathrm{biologia} / 2005 / \mathrm{tcc}$ _graciele_ timm.pdf>. Acesso em: 4 maio 2011.

WORLD HEALTH ORGANIZATION (WHO). Iron Deficiency Anaemia: Assessment, Prevention, and Control. A guide for programme managers. Geneva: World Health Organization, 2001. Disponível em: <http://whqlibdoc.who.int/hq/2001/ WHO_NHD_01.3.pdf>. Acesso em: 4 maio 2011.

Worldwide prevalence of anaemia 1993-2005: WHO Global database on anaemia. Geneva: World Health Organization, 2008. Disponível em: <http://apps.who.int/ iris/bitstream/10665/43894/1/9789241596657_eng.pdf>. Acesso em: 8 ago. 2017. 